\title{
The Ambi-room electric auto-control system of final Optics Assembly
}

\author{
Wang Liquan ${ }^{1}$, Jing Yukun ${ }^{1 \mathrm{c}}$, Feng Bing ${ }^{1}$, Xiang Yong ${ }^{1}$, Gao Zhongpen \\ ${ }^{1}$ Research Center of Laser Fusion CAEP, Mianyang, China \\ ${ }^{2}$ KYKY Technology CO., LTD, BeiJing, China \\ c email: wanglq1013@caep.cn , Correspondence: Tel: +86-18081247627
}

Keywords: Ambi-room; Final Optics Assembly; Purge Air Sweeping; Target Chamber; integrated optics module (IOM)

\begin{abstract}
This control system adopts Siemens S7-400 PLC to realize these functions of monitoring ambi-room pressure by the vacuum gauges and manipulating all vacuum valves in real time etc. The ambi-room control system belonged to unworthy man guard equipments has severe control request of "absolute safety", so that it must ensure safety and credibility in every operation flow: vacuum achievement, input dry clean air, keeping ambi-room pressure, dynamic state purge air sweeping etc. So safety design and manufacture is preferential to any other request, furthermore this idea passes through all control workflow. Because the user mutual interface is very friendly, the manipulation is simple and easy to learn for all assigned operators.
\end{abstract}

\section{Background}

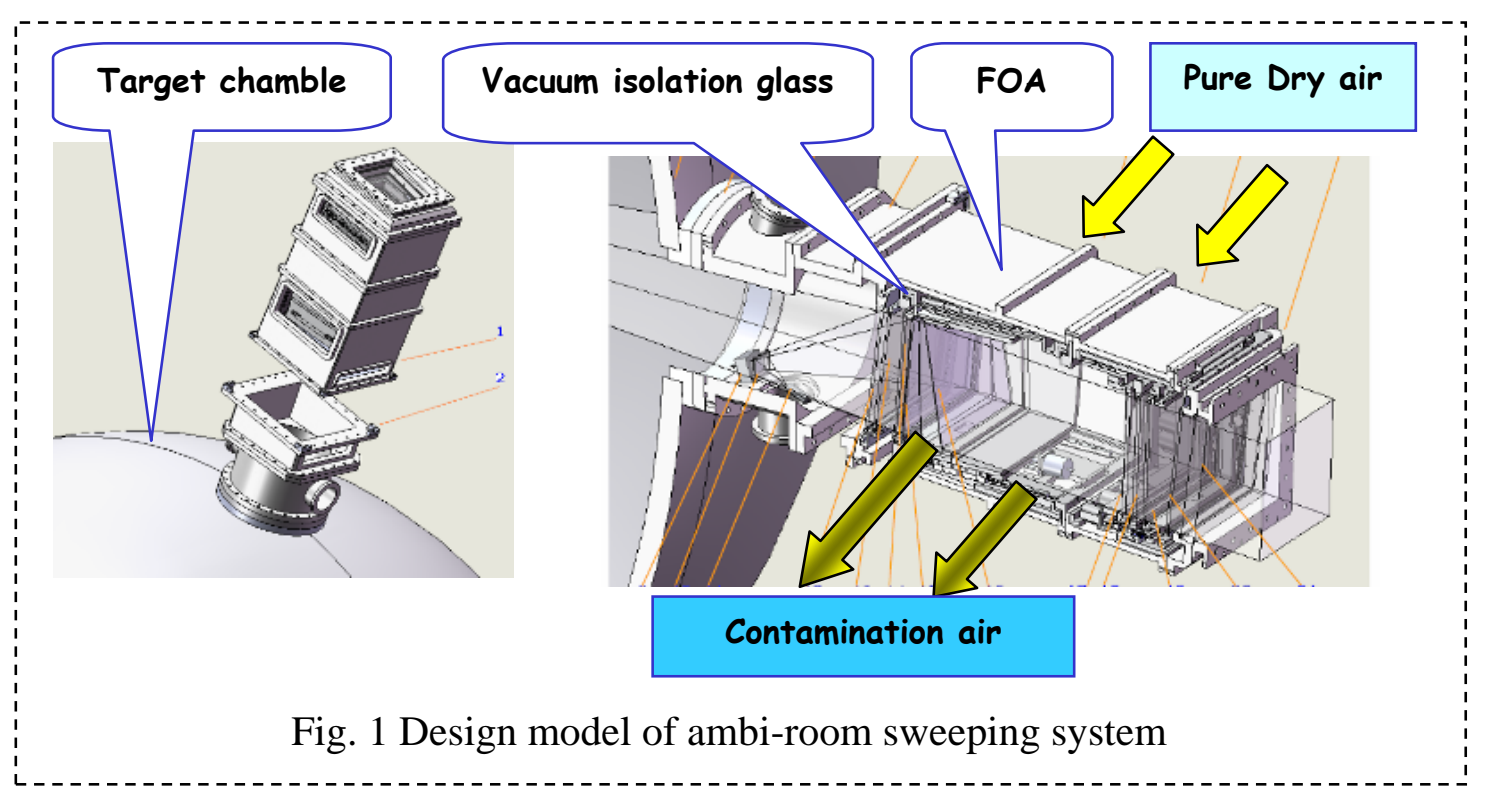

The final optics assemblies (FOAs) are the last element of the main laser system and the first of the target area systems. Each FOA contains one integrated optics modules (IOM) that incorporate beam conditioning, frequency conversion, focusing, diagnostic sampling, and debris shielding capabilities into a single compact assembly. These optics are shown in the Fig. 1. The same mechanical, optical, and beam control components that are used in the FOA at the target chamber are reproduced for a single beamline in the precision diagnostic system. Then, the ambi-room must chronically provide cleanness and rich oxygen atmosphere environment for FOA's long-term stability operation, which must guarantee the vacuum seal glass slice between FOA and vacuum target chamber through strictly control the pressure difference between the glass slice's two sides in order to confirm system security.

Because the FOA produces a great deal of spirit dissolve gum and other organic pollutant as soon as ICF laser driver high energy laser shoots for physical experiment, it will bring on quick deterioration of the FOA's inner cleanness environment. So, the ambi-room will also have the function for dry and clean air dynamic state blowing and sweeping, as well as fast gas displacement 
purge, in order to restore inner cleanness environment of the FOA by venting out contamination in a very short time. It can increase surface damage threshold value and extend life-span of the optics components in FOA, strengthen stability of the whole ambi-room system. Design model of ambi-room sweeping system as shown in Fig.1

\section{Basic function and constitution of Ambi-room system}

The single ambi-room system of FOA refers to the system with safety valves, which includes vacuum pipes, inlet pipes, vacuum valves, safety valves, vacuum gauges, vacuum pump, clean dry compressed air source, pressure sensors, pressure regulation valve and electrical control system etc. The single ambi-room's constitute diagram and electricity principle diagram see Fig. 2. Through controlling vacuum isolation valves, outlet valve, clean purge valve, flow regulation valve and inlet regulation valve, The ambi-room system can realize control the pressure of the inner chamber in real time, which makes the pressure difference between the ambi-room and target chamber in permission range in order to guarantee system safety operation. The system have three control processes in total, which are respectively vacuumizing room and keeping pressure process, inlet gas process, clean purge process.
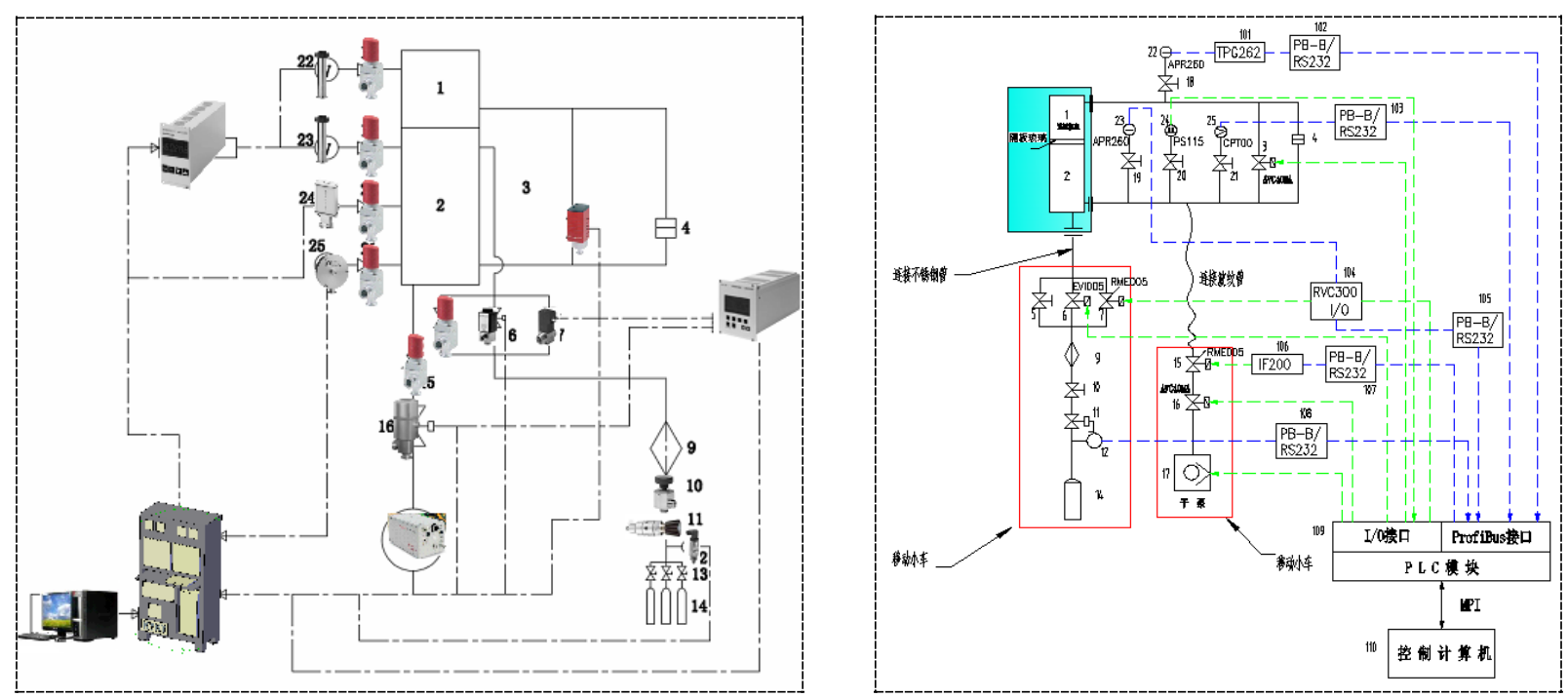

Fig.2 Concrete components and layout of single ambi-room system

\section{The electric control system}

According to the request of mechanical design, it adopts hard vacuum electromagnetism valve as he key control valve for vacuum gain and inlet gas, and take safety valve as key safety protection control valve for ambi-room and target chamber in permission pressure difference range. The vacuum gauges will be taken as dominating measure for ambi-room. Diaphragm vacuum gauge and electric resistance gauge and vacuum switch installed on stainless steel vacuum tubes can check different vacuum states of the chamber. The control system total structure see Fig. 3.

According to high stability request for the control objects and key driven components, PLC central control design has been redundancy backup, see Fig. 3. The local control system constituted of PLC inner ark and designated bottom operation will carry out system auto running and manual operation of the vacuum equipments including pumps and valves etc. without super supervision system, the ambi-room control system can still run in safety, because bottom PLC can normally continue inspecting each feedback point of the valves according to the program downloaded to PLC before. The process of PLC condition control vacuum system as shown in Fig 4. 


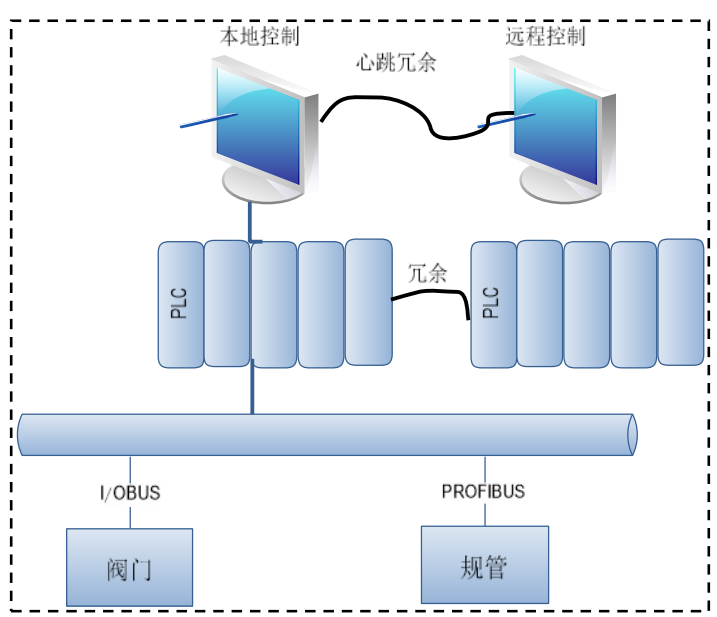

Fig.3 The control system total structure

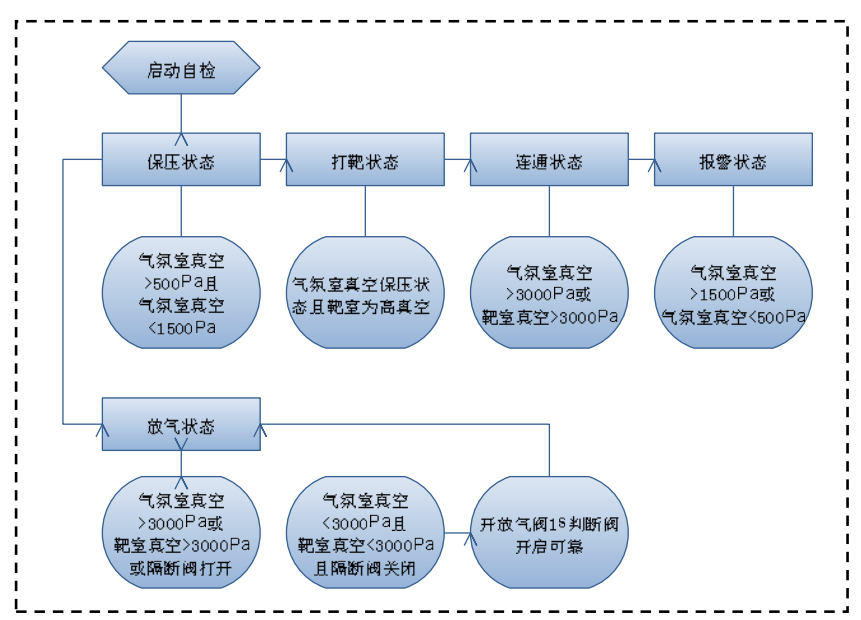

Fig.4 The process of condition control vacuum system

\section{Computer software interface}

With big screen takes charge of inspecting all states of the telecommunication performance components in the ambi-room control system. Combining mutually design for upper computer supervision interface and 3D mechanical design, it can directly show the vacuum dynamic states of the entity valves and chamber vacuum appearance, and display the different operation process of the ambi-room and target chamber. The control system adopts Siemens S7-400 PLC to handle vacuum gauges and electromagnetic valves to realize these functions such as vacuum surveillance, emergency measures, auto record process and important experiment data etc. Upper computer supervision interface and process data interface are shown Fig.5 and Fig. 6.

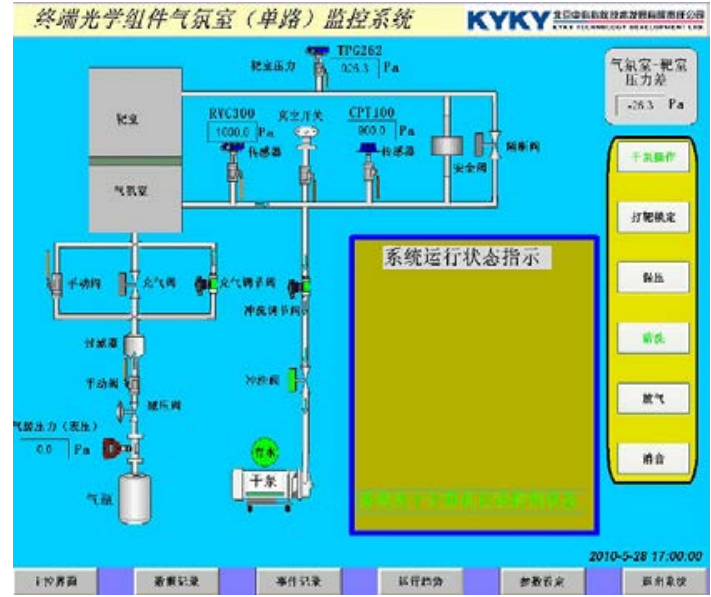

Fig.5 Upper computer supervision interface

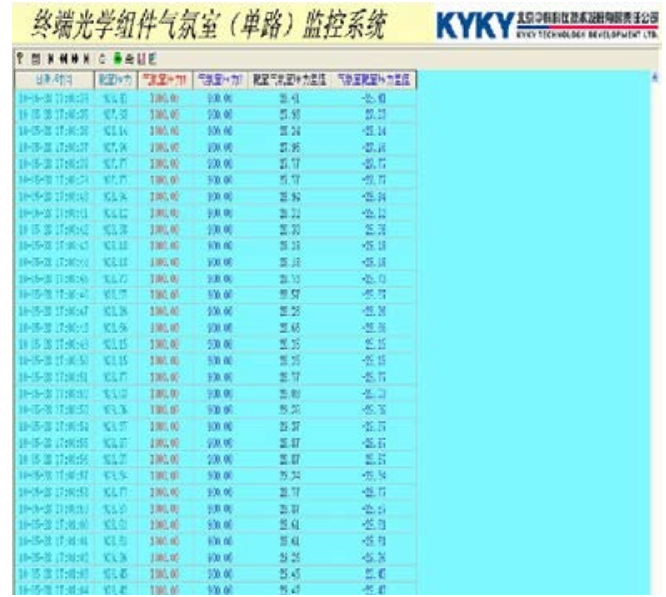

Fig. 6 control system process data show interface

\section{Result of the experiments}

After the ambi-room control system is installed on spot corresponding FOA, it carries out the verification experiment to validate the air dynamic state displacement function on the condition of the invariable ambi-room pressure. The ambi-room air displacement system adopts Pfeiffer company's oilless vacuum pump and PID control mode. It has made certain that the air output regulation valve's open degree is $89 \%$ when the air displacement flow flux reaches $2 \mathrm{~L} / \mathrm{s}$ for the flow flux requirement, and the ambi-room pressure still satisfy $1000 \mathrm{~Pa} \pm 500 \mathrm{~Pa}$ for the safety requirement. The curve achieved in Experiment is shown in Fig. 7. 


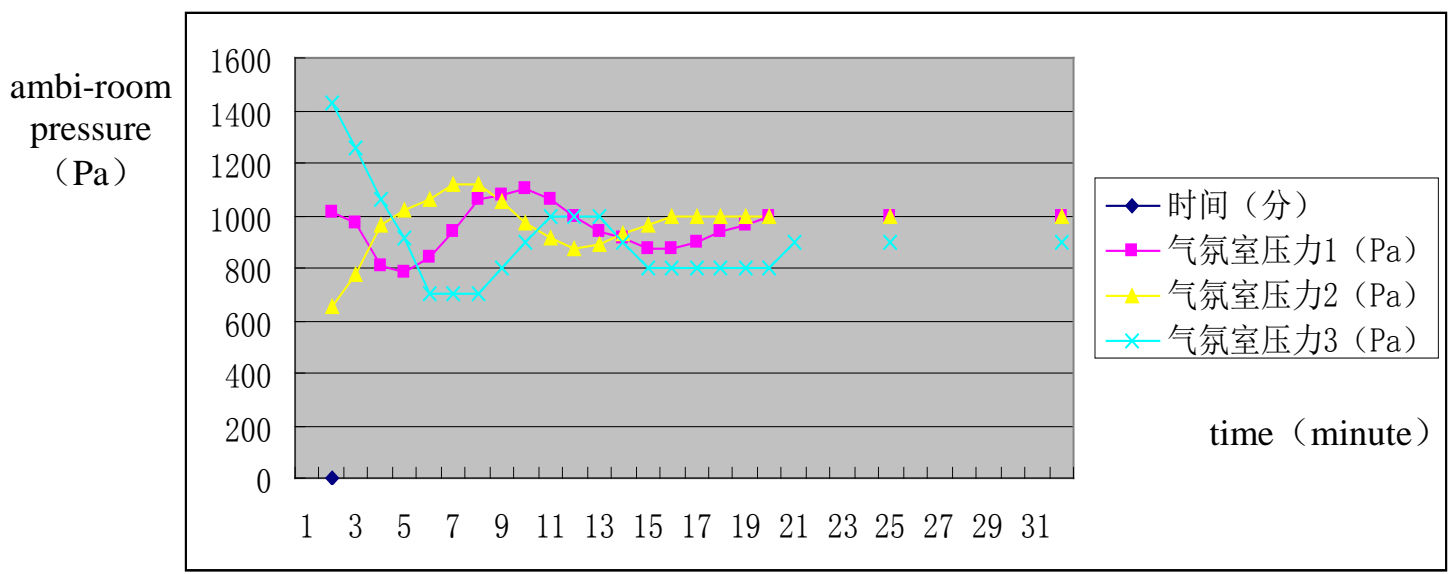

Fig. 7 The pressure curve of the air dynamic state displacement

\section{References}

[1] R. Gavaggio, P. Strubin: "Examples of Applications of. Industrial Control Systems (PLCs) For Vacuum. Equipment", Proceedings ICALEPCS'97[C], 3-7th, November 1997

[2] T. M. Stephen, B. Van Zyl, R. C. Amrne, "Degradation of vacuum-exposed $\mathrm{SiO}_{2}$ laser windows,” SPIE vol. 1848, pp. 106-110 (1992).

[3] M. Shaw, W. Williams, R. House, C. Haynam, "Laser performance operations model,” Opt. Eng., 43(12) 2885-2895 (2004).

[4] J. Paisner and J. R. Murray, “Overview of the National Ignition Facility Project," Proc. SPIE Conf. Solid-State Laser Apps. To ICF (Monterey, CA, 1998).

[5] L. J. Lagin et al., "The Overview of the National Ignition Facility Distributed Computer Control System", Conf C011127 (2001) TUAP001 